\title{
Evaluation of Silk Inverse Opals for "Smart" Tissue Culture
}

\author{
Peter Tseng, ${ }^{\dagger}$ Siwei Zhao, ${ }^{\ddagger}$ Annie Golding, ${ }^{\ddagger}$ Matthew B. Applegate, ${ }^{\dagger}$ Alexander N. Mitropoulos, ${ }^{\ddagger}$

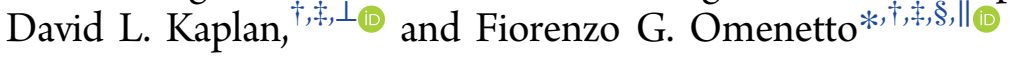 \\ ${ }^{\dagger}$ Silklab, Tufts University, 200 Boston Avenue, Suite 4875, Medford, Massachusetts 02155, United States \\ ${ }^{\ddagger}$ Department of Biomedical Engineering, ${ }^{\S}$ Department of Electrical and Computer Engineering, "Department of Physics, and \\ ${ }^{\perp}$ Department of Chemical Engineering, Tufts University, Medford, Massachusetts 02155, United States
}

\section{Supporting Information}

ABSTRACT: Visually tracking the subtle aspects of biological systems in real time during tissue culture remains challenging. Herein, we demonstrate the use of bioactive, cytocompatible, and biodegradable inverse opals from silk as a multifunctional substrate to transduce both the optical information and cells during tissue culture. We show that these substrates can visually track substrate degradation in various proteases during tissue digestion and protein deposition during the growth of mesenchymal stem cells. Uniquely, these substrates can be integrated in multiple steps of tissue culture for simple-to-use, visual, and quantitative detectors of bioactivity. These substrates can also be doped, demonstrated here with gold nanoparticles, to allow additional control of cell functions.
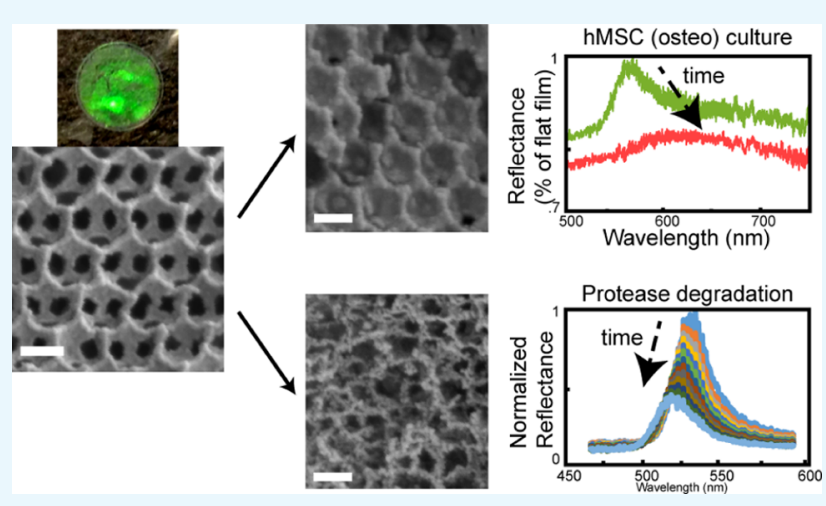

\section{INTRODUCTION}

Tracking the changes in biological systems during tissue culture provides insight into the dynamic changes and optimization of outcomes. This insight allows the systems to play a more critical role in medicine, pharmacology, and fundamental research. Biosensing schemes designed to facilitate "smart" cell and tissue culture traditionally exploit a variety of biosignals, including electrical properties ${ }^{1-5}$ (impedance or electrical potential), mechanics, ${ }^{6-9}$ and secreted biomolecules, such as extracellular matrix (often using interferometry). ${ }^{10-13}$ Optical biosensors are among the least invasive of the above modes of detection, which can potentially provide direct, visual feedback on cell activity without the need for additional external interrogation. Examples of biosensors include diffraction gratings, opaline lattices, and Bragg reflectors, which have been used to detect protease activity, biomolecules (both concentration and adsorption), and biochemistry. ${ }^{10,14-17}$

The majority of these optical structures are based on silicon/ porous silicon or synthetic polymers, which are formed by either traditional, time-consuming microfabrication techniques (in the case of silicon) or templating (for polymeric materials). In general, these structures must be chemically postprocessed and doped to interface with living tissue. For example, to study protease activity, substrates must be modified or infiltrated with proteins, such as zein or gelatin, to obtain a bioresponsive surface and substrate. ${ }^{14,15}$ Of note, in the majority of these optical biosensors, the optical effect is not overt and requires external interrogation, such as a spectrometer. ${ }^{18}$ Generally, such substrates are also inert sensors and cannot be easily modified to interact with the biological systems they measure (i.e., provide growth factors, enzymes, or engineered stimuli). In contrast to silicon and synthetic polymers, biopolymers are a potentially simpler and "greener" material counterpart due to their native biocompatibility and renewable sourcing. Recent efforts have focused on engineering such biomaterials to possess controlled three-dimensional micro- and nanostructures. For example, chitosan has been formed into mesoscale inverse opal microstructures, through which cells can infiltrate and grow, ${ }^{19}$ and optically responsive nanostructured inverse opals have been formed from biopolymers keratin and silk fibroin. ${ }^{16,20,21}$ In addition to providing a colorimetric response, such substrates are easily doped with biomolecules and inorganic compounds, ${ }^{22}$ enabling more functional capabilities than those of traditional silicon-based sensors.

In this article, we utilize inverse opals formed directly from native or doped silk protein to form multifunctional substrates capable of passive sensing of protease activity (commonly used to digest tissues or release cells from a substrate) and biological substrate remodeling (commonly generated by fibroblasts or stem cells). The sensing is colorimetric and visible to the eye, with additional potential for cell activation/disruption via direct doping of the substrate. Such visual detection can be achieved in situ, potentially allowing direct transduction from active substrates without having to utilize fluorescence detection methods that often require fixation or destruction of cells along

Received: October 17, 2016

Accepted: December 27, 2016

Published: February 10, 2017 


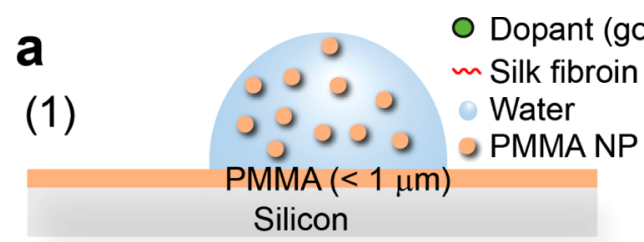

(2)

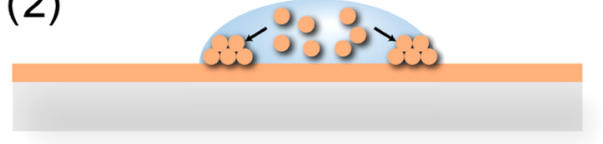

b

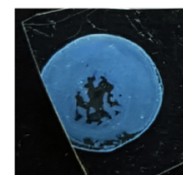

Fill:

Air

$$
\Lambda=250 \mathrm{~nm}
$$

d

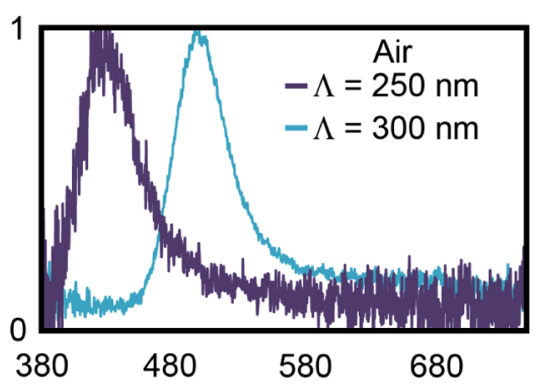

f

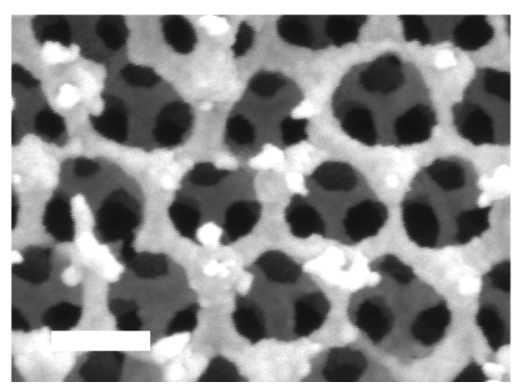

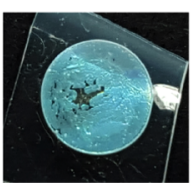

$\Lambda=300 \mathrm{~nm}$

e

C

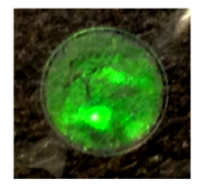

$\Lambda=260 \mathrm{~nm}$

Fill: Water

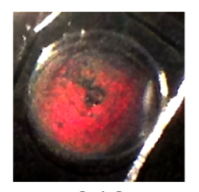

$\Lambda=310 \mathrm{~nm}$

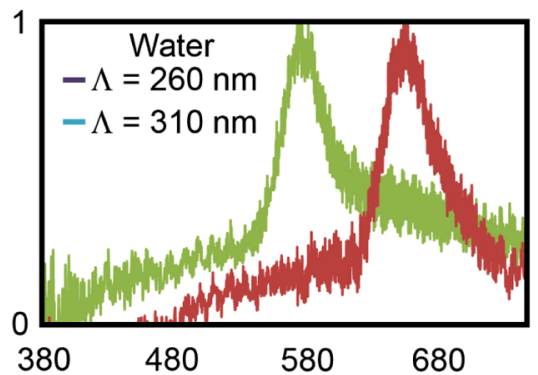

g

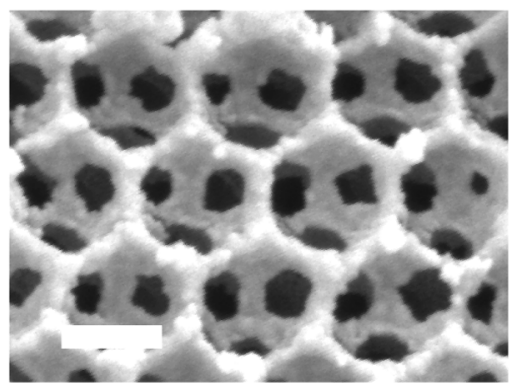

Figure 1. Doped-silk inverse opals for smart cell culture. (a) Fabrication process for inverse opals. (b) Silk inverse opals in air formed from 250 and $300 \mathrm{~nm}$ PMMA beads. (c) Silk inverse opals in water. (d) Normalized reflectance in air for silk inverse opals of 250 and $300 \mathrm{~nm}$. (e) Equivalent reflectance in water. (f, g) Open-faced inverse opals formed from 250 and $300 \mathrm{~nm}$ beads. Scale bars are $200 \mathrm{~nm}$.

with the use of optical systems for their excitation and detection. The inverse opals formed from silk enable effective, simple biosensors and functional substrates for "smart" biological tissue culture platforms. Silk fibroin, due to its mechanical strength and low surface tension, has found applications in drug delivery, ${ }^{23}$ tissue engineering, ${ }^{24}$ optics, ${ }^{16}$ electronics, ${ }^{25,26}$ and so on. The use of silk in tissue culture has numerous notable advantages, including the facilitation of cell growth and proliferation without dopants or postprocessing, ${ }^{27}$ the ability to stabilize dopants (such as enzymes and growth factors), 22,28,29 and tunable biodegradability when exposed to proteases. $^{30,31}$ These advantages have enabled the use of silk biomaterials in a diverse set of tissue culture applications ranging from stem cell differentiation ${ }^{32}$ to tissue formation ${ }^{33}$ and mechanotransduction studies. ${ }^{34}$ Previously, silk inverse opal substrates have been used to track humidity, ${ }^{35}$ as a substrate for cell growth $(10 \mu \mathrm{m}$ pores $),{ }^{36}$ and to study analytebinding events. ${ }^{37}$ Here, we study the potential of such substrates as an active biointerface to optically and quantitatively track the growth/remodeling of cells and the digestive state of tissue when subjected to protease. Furthermore, we demonstrate that such substrates can be easily doped to provide functional interfaces with biological systems, thus providing an additional layer of control that is not easily achieved in traditional, silicon-based, or other inorganic substrate materials.

\section{RESULTS AND DISCUSSION}

Silk inverse opals were formed via capillary infiltration into a colloidal template. Circular 4-6 mm inverse opal templates were formed via evaporative drying of poly(methyl methacrylate) (PMMA) beads (Phosphorex, 1\% suspension of 250 $\mathrm{nm}$ and $300 \mathrm{~nm}$ diameter beads) on a PMMA-spun silicon substrate. Subsequently, silk solution (6\%) was cast, alongside optional dopants, onto template substrates and slowly dried over $24 \mathrm{~h}$ to form inverse opal structures cast within a silk film (Figure 1a). Silk films dried via this approach were primarily amorphous and water-soluble. To render inverse opals waterinsoluble (and thus viable for tissue culture), the films were water-annealed for a minimum of $4 \mathrm{~h}$ to transform the silk 
a

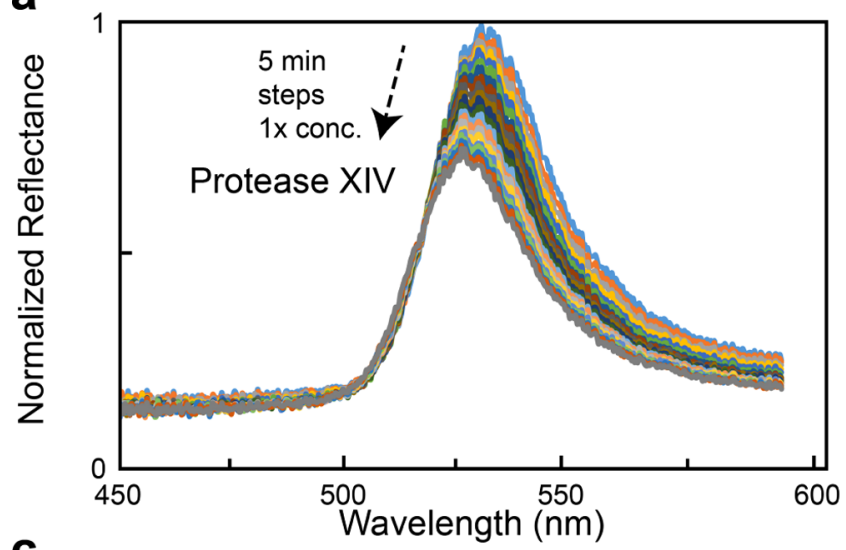

C

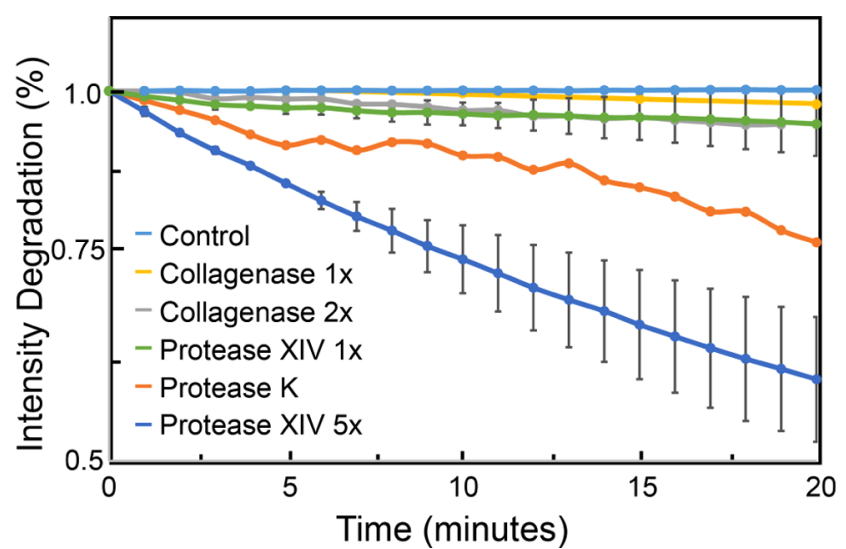

b
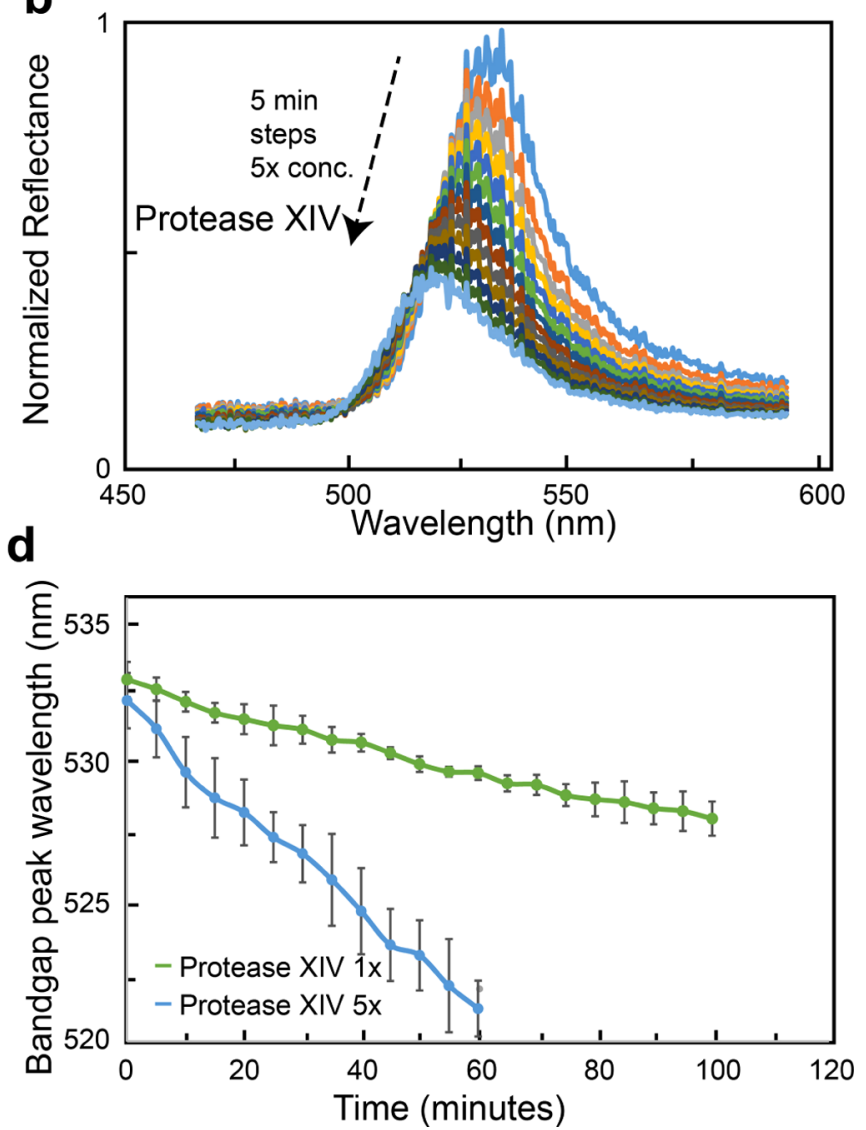

Figure 2. Silk inverse opal degradation in response to protease. (a) Reflectance over time during opal incubation in $1 \times$ protease XIV. (b) Reflectance over time during incubation in $5 \times$ protease XIV. (c) Comparison of reflectance degradations in a variety of proteases. (d) Bandwidth peak wavelength over time during incubation in protease XIV.

fibroin into water-insoluble $\alpha$-helix and $\beta$-sheet conformations. The PMMA template was then dissolved overnight in acetone before being solvent-exchanged to water first via immersion in isopropanol and then with the slow addition of water.

The band gap maximum wavelength of inverse opal structures can be estimated using the equation ${ }^{38}$

$$
\lambda_{\max }=1.633 D\left[\varphi n_{\text {walls }}+(1-\varphi) n_{\text {voids }}\right]
$$

where $D$ is the periodicity of the structure, $\varphi$ is the fill percentage/porosity ( 0.06 for typical inverse opals), and $n$ is the refractive index of the solid $\left(n_{\text {silk }} \sim 1.6\right)$ and the void regions $\left(n_{\text {air }}=1\right.$ and $\left.n_{\text {water }}=1.33\right)$ of the inverse opals. Increases in the PMMA bead size and reduction in the index difference between the walls and the voids led to a redshift in the band gap peak wavelength of the structure. The optical response of the silk inverse opals was probed using a fiber-coupled spectrometer (Ocean Optics USB2000+; Dunedin, FL) in a reflection geometry. In air, the inverse opals possess peak reflectance in the blue $(\lambda \approx 410 \mathrm{~nm})$ and teal $(\lambda \approx 500 \mathrm{~nm})$ regions that correspond to the stopband of the structures with lattice periodicities of 250 and $300 \mathrm{~nm}$, respectively. When immersed in water or culture medium $(n=1.33)$, the inverse opals lightly swelled and the index contrast changed with redshifting of the reflective peaks to $\lambda \approx 575$ and $660 \mathrm{~nm}$ (Figure $1 \mathrm{~b}-\mathrm{e})$. The inverse opals formed together from the same silk treatments will have minor variations in reflective peaks $( \pm 2$ $\mathrm{nm}$ ), although reflectance peaks from the samples formed from different batches can vary more significantly $( \pm 20 \mathrm{~nm})$ due to silk age and crystallization. The crystallinity of silk fibroin affects its swelling when immersed, which is primarily responsible for the batch-to-batch variation in the peak reflectance center wavelength. The silk inverse opal structures could be visualized through scanning electron microscopy (SEM) that shows a three-dimensional face-centered cubic lattice of voids (Figure 1f,g). To visualize the lattice of these nanostructures, we generated "open-faced" structures via reflow of the PMMA/photoresist sacrificial layer.

A major aspect of tissue culture is enzymatic digestion: a step used for cell passage, to isolate primary cell lines from tissue; extract proteins, DNA, and other biomolecules; and assess substrate remodeling during tissue regeneration. We first studied the potential of undoped silk inverse opals as sensors for protease activity and tissue digestion (Figure 2). Silk possesses cleavage sites for a majority of proteases, such as collagenase, proteinase $\mathrm{K}$, protease XIV, matrix metalloproteinases, and others. ${ }^{30}$ The temporal response of the inverse opals to different concentrations of protease XIV (commonly used to nonspecifically dissociate tissues) was first assessed. In general, we would expect two responses during a protease etch (Figure S1): (1) a blueshift in the band gap peak wavelength due to the loss of high-index material in the inverse opal wall and (2) a reduction in the reflectance due to the nonisotropic etching reducing the quality of the inverse opal network. This expected response was observed at both high and low concentrations of protease XIV (Figure S2, $N=4$ ), indicating the capability of the opals to optically transduce and visibly resolve digestion rate. 

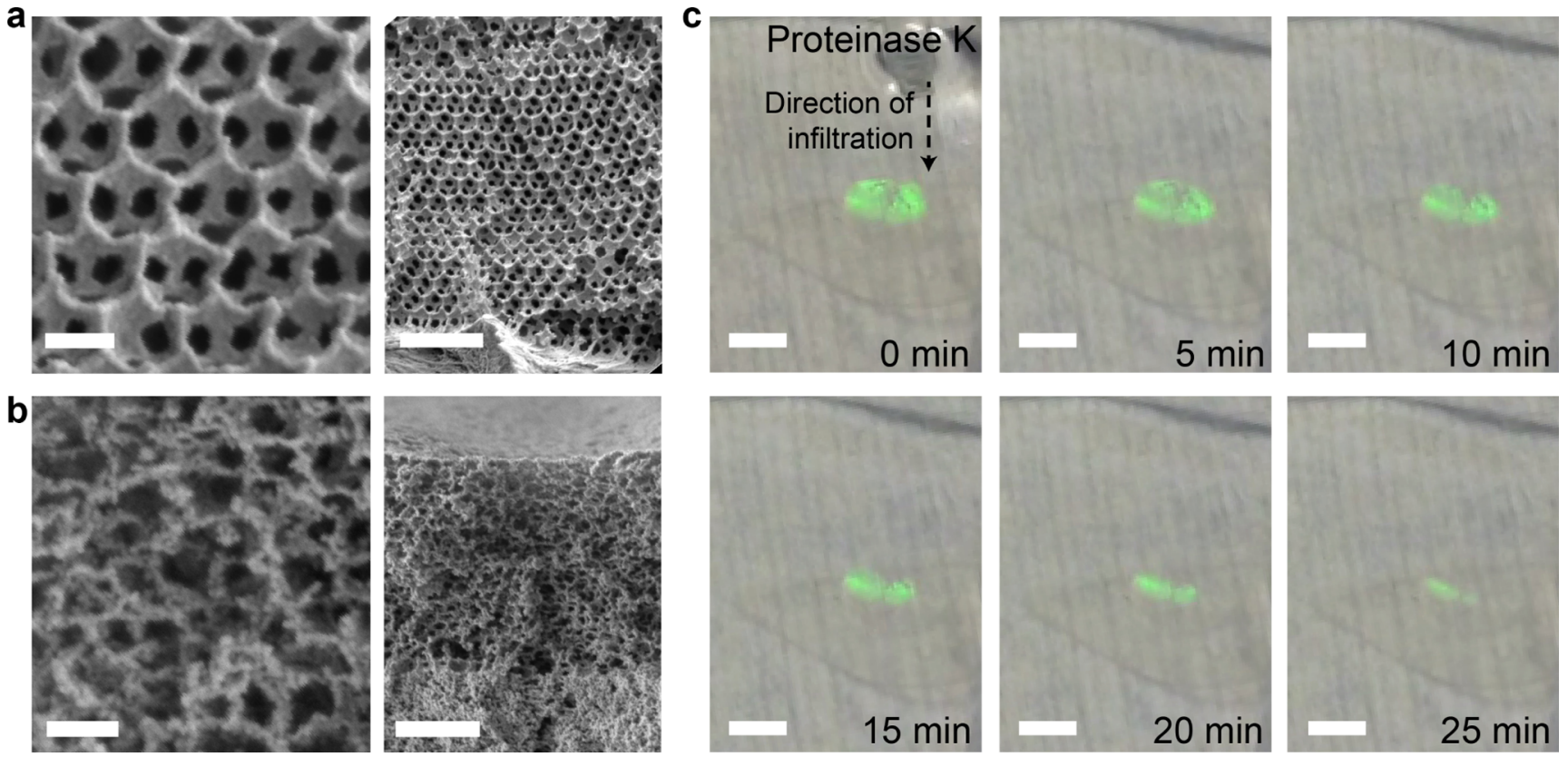

Figure 3. (a) Cryofractured silk inverse opal. The thickness of the opal structure is approximately $4-5 \mu \mathrm{m}$. Scale bar is $200 \mathrm{~nm}$. (b) Cryofractured protease XIV-degraded opal. The resultant structure is different from that of a standard opal and possesses an increase in porosity and nanostructure collapse. The thickness was approximately $2 \mu \mathrm{m}$, confirming partial structure collapse during drying. Scale bar is $1 \mu \mathrm{m}$. (c) Degradation of inverse opal ( $\sim 2 \mathrm{~mm}$ wide) color during asymmetric addition of proteinase $\mathrm{K}$. The protease was added above the structure, and the color degradation was observed over time. Scale bar is $2 \mathrm{~mm}$.

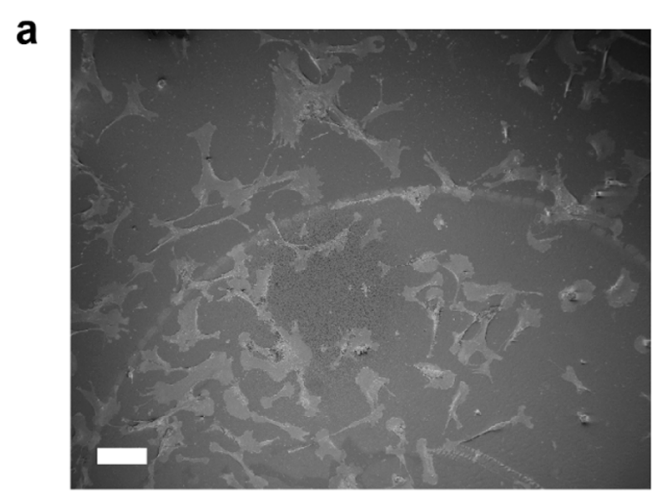

C

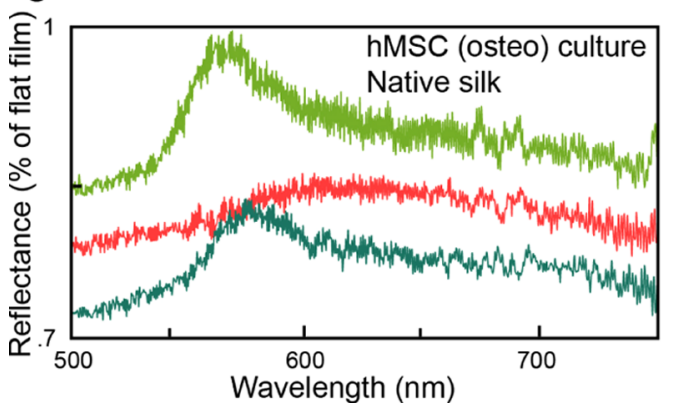

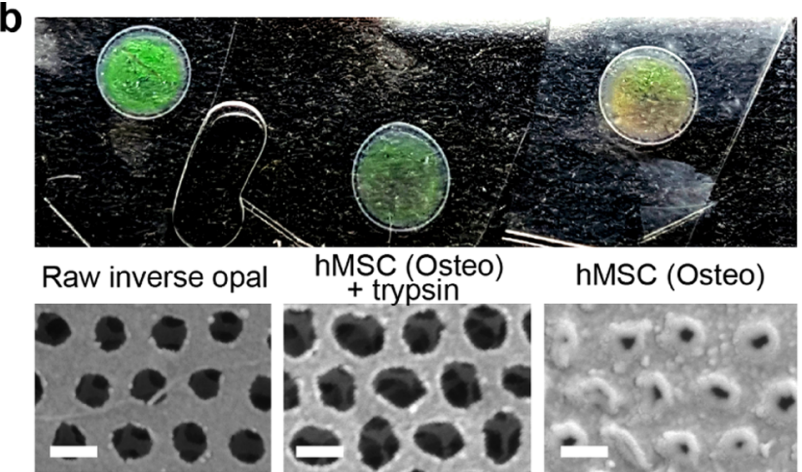

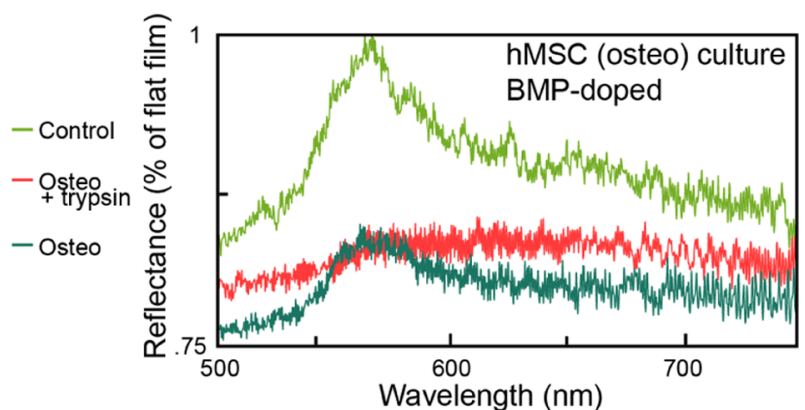

Figure 4. Biological response of silk inverse opals. (a) Fibroblast growth on inverse opal topography. Cells generally do not prefer flat silk over inverse opal silk topography. Scale bar is $200 \mu \mathrm{m}$. (b) Opal response during tissue culture. Growth of osteogenically differentiated human mesenchymal stem cell (hMSC)-yielded substrates with adsorption of protein/mineral. These deposits could be removed (included partial etching of silk) with trypsin. Scale bars are $200 \mathrm{~nm}$. (c) Reflectance comparison for different inverse opals, one set doped with BMP and the other undoped. Samples with cell growth exhibited broadening of the band gap and a redshift, and trypsinization of the samples resulted in partial recovery of the initial opal response.

Subsequently, the reflectance degradations of control samples (in water) and those incubated in a variety of proteases, including two concentrations of collagenase, protease XIV, and proteinase $\mathrm{K}$, were compared. As expected, increasing the concentrations of either collagenase or protease XIV led to high rates of reduction in reflectance intensity. The observed colorchange dynamics was different for different enzymes used (i.e., Proteinase $\mathrm{K}>$ protease $\mathrm{XIV}>$ collagenase, indicating that 
a

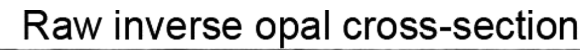

b

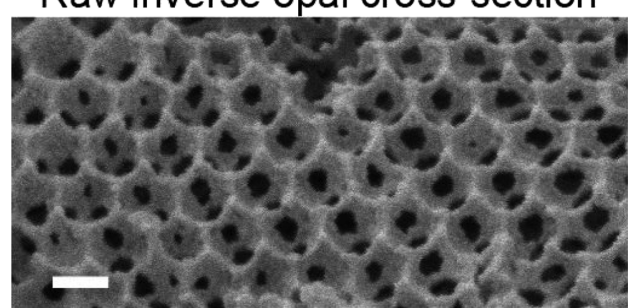

b hMSC (osteo) Position 1 cross-section

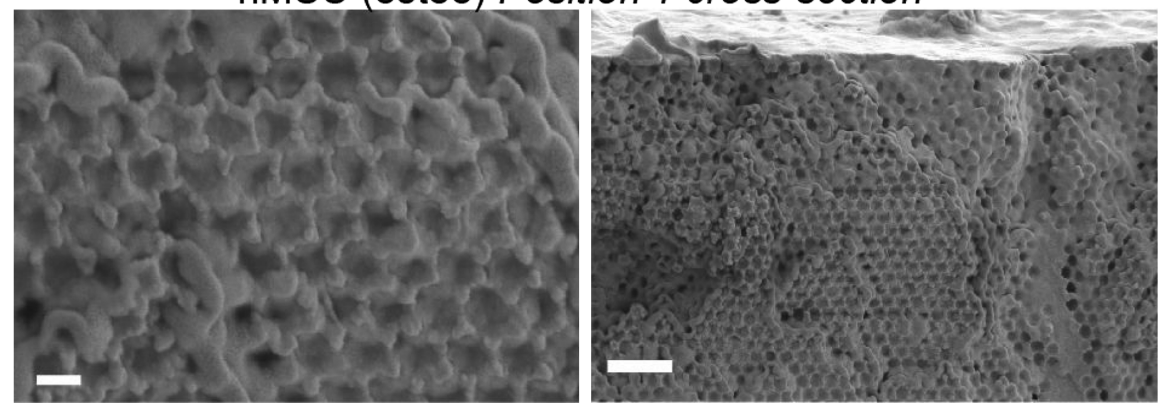

C

hMSC (osteo) Position 2 cross-section
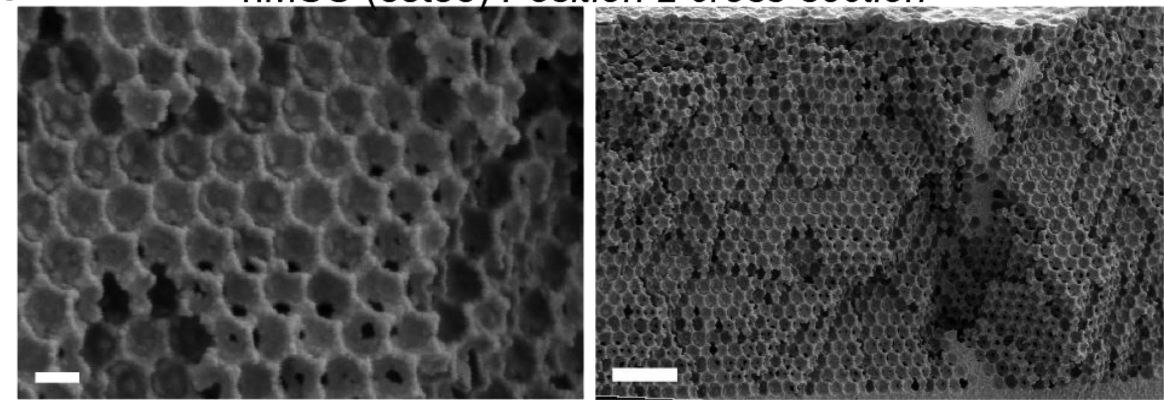

Figure 5. Cryofractured cell-treated inverse opals. (a) Raw inverse opal cross section with a porous structure. Scale bar is 200 nm. (b, c) Cryofractured inverse opals at two different positions (near the center of the opal and toward the edge). Protein adsorbed to a thickness of 30-70 $\mathrm{nm}$ depending on the position and depth. Left scale bars are $200 \mathrm{~nm}$, and right scale bars are $1 \mu \mathrm{m}$.

proteinase $\mathrm{K}$ degrades the substrate at the highest rate). This result matches that of the previous studies on silk degradation ${ }^{30}$ and suggests that these structures can track proteinase K- and protease XIV-based digestion mixtures more easily than the collagenase-based ones. The protease variability in the response also suggests that these structures are more suited for sensing the activity of known protease cocktails rather than the proteolytic activity of biological cells (which secrete complex mixtures of proteases). The response was close to linear for the protease concentrations and exposure times used in our experiments. For example, the reflective peak frequency shifted $3 \mathrm{~nm}$ for $1 \times$ concentration of protease XIV in $60 \mathrm{~min}$, whereas it shifted $12 \mathrm{~nm}$ for $5 \times$ concentration of protease XIV in the same amount of time (results were similar for absorbance studies). The results suggest that the inverse opal biosensors can be used both as a transducer of silk degradation for basic studies and as a directly visible marker of protease activity and digestion competition during the tissue dissociation steps.

During degradation of the opals, the wavelength shifts are of the order of $10 \mathrm{~nm}$. From theory, changes in the inverse opal fill factor of 0.01 yield a shift of $\sim 1 \mathrm{~nm}$, implying a higher observed shift than expected. ${ }^{38}$ This difference is likely due to a collapse or shrinking of the inverse opal structure during protease digestion. SEM images of cryofractured inverse opal structures (Figure 3) before and after the protease digestion show both random disruption of the lattice and an associated increase in porosity. The thickness of protease-etched structures was less than half of that of nonetched (control) structures, indicating a significant collapse during the digestion and drying process.

The visible response of the silk inverse opals to proteases was demonstrated using the time-lapse imaging of the structures during the addition of proteinase $\mathrm{K}$ by adding $20 \mu \mathrm{L}$ of protease on the opal structures and imaging the response over $30 \mathrm{~min}$. The inverse opal steadily degraded and the structural color slowly disappeared, indicating the ability to visibly distinguish response without the aid of external interrogation with a spectrometer.

Because of the biocompatibility of silk fibroin, fetal foreskin fibroblast cells were cultured directly on the inverse opals to study their effect on cell adhesion and patterning. Inverse opals formed in a standard format (on PMMA) exhibited a minor effect on cell patterning (Figure 4a). Although large-cavity holes were found to modulate cell adhesion on other surfaces, this phenomenon was not observed for the inverse opals formed from 250 and $300 \mathrm{~nm}$ beads. Interestingly, silk nanostructured surfaces (formed via PMMA bead formation on photoresist and subsequent induction of heavy reflow, as described in the Experimental Section) modulated cell adhesion consistently with that reported in the literature ${ }^{39}$ (Figure S4b), leading to an increase in the local coverage of cells above the "rough" nanostructured region. 
The potential of silk inverse opals to track substrate remodeling during cell growth was studied. Osteogenic differentiating mesenchymal stem cells were utilized to study the growth and release of growth factors, matrix metalloproteinases, insoluble matrix, and mineralization, which combine to remodel existing substrates. ${ }^{40}$ These cells are also important in pharmacology and medicine for regenerative medicine. hMSCs were seeded onto four green (peak reflectance $\lambda \approx 500 \mathrm{~nm}$; lattice constant $300 \mathrm{~nm}$ ) inverse opal substrates. Two of the opals were doped with bone morphogenic protein (BMP-2) and allowed to grow in differentiation medium over 24 days to induce osteogenesis (Figure S4c). At 24 days, a redshift and dimming in the inverse opal response was noted. Half of the samples were fixated in formaldehyde and the other half were trypsinized for $10 \mathrm{~min}$ (followed by cold shock for $1 \mathrm{~h}$ ) to remove proteins from the surface. These samples were then compared with untreated inverse opals using optical imaging and a spectrometer. The samples incubated with cells exhibited a significant redshift of up to $30 \mathrm{~nm}$ alongside a noticeable loss in luster that was visible to the eye (Figure 4). Trypsinized samples exhibited partial recovery of the original inverse opal behavior, indicating that biomolecules and mineralization entrapped by insoluble and adsorbed proteins (likely a high percentage of collagen) were likely the sources of the redshift and the associated dimming of the inverse opal response. To expose the surface for SEM imaging, the cells were gently scraped from the substrate and the samples were dried by critical-point drying, followed by the deposition of a thin layer of gold for imaging. Mesenchymal stem cell cultures occlude nano-holes over time ${ }^{41}$ (reducing their visibility), and a similar response was noted on the substrates prepared here (Figure $4 \mathrm{~b}$ ). Trypsinized surfaces did not exhibit this effect, suggesting that the trypsinization process removed the occlusions within the opal lattice.

The samples were also cryofractured to observe the internal structure of the nanostructured lattice (Figure 5). The images revealed significant filling within the inverse opal with an observable deposition of biomaterial on the internal surface of the opal voids at a depth between 20 and $50 \mathrm{~nm}$. This reflects a change in the fill factor/porosity of the opal from 0.06 up to a range between 0.15 and 0.4 . This is associated to a redshift of $\sim 40 \mathrm{~nm}$, which suggests the ability of silk inverse opals to optically track the dynamic changes in substrate remodeling during tissue culture.

The ease of dopability of silk (i.e., with biomolecules, ${ }^{22}$ gold nanoparticles, ${ }^{16}$ quantum dots, ${ }^{42}$ etc.) additionally provides excellent opportunities in creating a programmable and interactive substrate for use during cell culture, which is otherwise not possible with traditional inorganic materials. To demonstrate this concept, the silk inverse opals were formed by including gold nanoparticles (Au-NP: diameter $\sim 10 \mathrm{~nm}$ ) in the silk solution (Figure S5). These particles absorb strongly at their plasmonic resonant frequency, converting light into heat. ${ }^{16}$ Such functional substrates were excited with a green laser $(532 \mathrm{~nm})$, increasing the local temperature to $70{ }^{\circ} \mathrm{C}$ under aqueous conditions, as measured via infrared thermography. When exciting substrates while growing cells, it was possible to target specific areas of the culture, such as patterned structures, and affect cell fate locally, inducing local cell death by directing light on the culture surface. These simple experiments hint at the versatility of these material systems that can enable a smart culture system with built-in multifunctionality and programmable function.
In conclusion, we have explored the potential of inverse opal structures derived from silk biopolymers as a platform material for "smart" tissue culture. The native properties of such structures (including biocompatibility and biodegradability) allow these systems to be used as sensors during multiple phases of tissue culture, including growth, digestion steps, and remodeling. Such a platform can also be straightforwardly doped, allowing for an additional layer of interaction and control in such devices. Silk inverse opal sensors display a rapid visual response in the presence of a variety of proteases used in tissue digestion, whereas their biocompatibility (in either undoped or doped format) facilitates the growth and remodeling of surfaces during cell culture. These devices present a compelling alternative to conventional inorganic materials, offering a suite of potential applications in medicine, pharmacology, and fundamental research.

\section{EXPERIMENTAL SECTION}

Preparation of Silk. Silk fibroin extraction protocols are detailed elsewhere. ${ }^{43}$ Briefly, silk cocoons were cut and boiled for $30 \mathrm{~min}$ to extract sericin. Silk fibers were dissolved in $9.4 \mathrm{M}$ $\mathrm{LiBr}$, dialyzed against deionized water, and centrifuged to form a clear, $7 \%$ solution of extracted fibroin.

Preparation of Silk Inverse Opals. Silicon wafers were cleaved and spun with a thin layer of PMMA (495PMMA C, Microchem) or positive photoresist (S1813). PMMA bead solution $(3.5 \mu \mathrm{L}, 250$ and $300 \mathrm{~nm}$, Phosphorex) was dropped onto the substrates, which are subsequently baked on a level hot plate at $85{ }^{\circ} \mathrm{C}$. Reflow can be induced in structures by additional baking at $95^{\circ} \mathrm{C}$ after this step (1-5 min, depending on the desired level). Silk solution (7\%, doped with BMP-2 or gold nanoparticles) was infiltrated above PMMA opals and allowed to dry over $24 \mathrm{~h}$ to achieve a desired silk film thickness of $100 \mu \mathrm{m}$. After drying, the samples were water-annealed under vacuum with a vial of water for $4 \mathrm{~h}$ and subsequently dropped in acetone over $24 \mathrm{~h}$ to etch the PMMA network. The samples were finally exchanged to water by slow washing with isopropanol and subsequently water. The etched inverse opals were stored in $10 \%$ ethanol or isopropanol to prevent bacterial growth. Dry inverse opals were formed by first transferring the substrates from water into $100 \%$ ethanol and finally criticalpoint-dried (Tousimis).

Inverse Opal Optical Characterization. The optical response of the inverse opals was analyzed by a probe spectrometer (USB2000; Ocean Optics). For aqueous inverse opals, the working solutions were dropped onto a glass slide, and a single-cut inverse opal was placed into the solution, covered with a glass coverslip and edges dried. To prevent evaporation, the coverslip edges were covered with a sized, square PDMS stencil. The samples were finally held underneath the probe, and spectra were captured and stored for analysis.

SEM Preparation. Cleaved samples were prepared via cryofracture. All samples were sputtered with a $10 \mathrm{~nm}$ thick layer of gold before being imaged using a Leica Ultra55 imaging tool.

Protease Degradation. Proteinase K from Tritirachim album (Sigma), protease XIV from Streptomyces griseus (Sigma), and collagenase (Worthington) stock solutions were prepared to 400,50 , and $1500 \mathrm{U} \mathrm{mL}^{-1}$, respectively. For time-lapse protease degradation experiments, $20 \mu \mathrm{L}$ of proteinase $\mathrm{K}$ stock solution was added at a location close to the silk inverse opal and imaged using a CCD camera. Working protease concentrations (Proteinase $\mathrm{K}: 4 \mathrm{U} \mathrm{mL}^{-1}$, protease XIV: $1 \times=$ 
$2 \mathrm{U} \mathrm{mL}^{-1}$, and collagenase: $1 \times=150 \mathrm{U} \mathrm{mL}^{-1}$ ) were applied to silk inverse opals, and opal spectra were analyzed over time using the spectrophotometer.

Cell Culture. Mouse fibroblasts and hMSCs (adiposederived) were isolated as described in existing protocols. ${ }^{44}$ Before cell culture, silk inverse opals were transferred into $70 \%$ ethanol for sterilization and slowly equilibrated to phosphatebuffered saline over several hours. The samples were finally transferred into the respective culture medium for $30 \mathrm{~min}$ before cell seeding. For fibroblast cell culture, cells were grown over $48 \mathrm{~h}$ before being fixed in $4 \%$ formaldehyde over $15 \mathrm{~min}$. The samples were stained for phalloidin (Sigma) and imaged by fluorescent microscopy before being transferred into ethanol for critical-point drying and SEM imaging. For hMSC cultures, cells attached to silk substrates were grown in osteogenic medium (Stempro, Invitrogen) for 24 days, with the medium refreshed once a week. Upon completion of growth, the samples were split and processed by either: (1) trypsinization for $10 \mathrm{~min}$ before cold shock at $4{ }^{\circ} \mathrm{C}$ for $1 \mathrm{~h}$ to remove cells and protein or (2) fixation in $4 \%$ formaldehyde over $15 \mathrm{~min}$.

Laser Heating. Gold nanoparticle-doped samples were excited using a $\mathrm{Nd}: \mathrm{YVO}_{4}$ laser (Verdi V-10, $532 \mathrm{~nm}$ ) and characterized using a thermal camera (SC-600, FLIR). The substrates were allowed to equilibrate over $5 \mathrm{~min}$ so that the sample temperature could be stabilized and imaged.

\section{ASSOCIATED CONTENT}

\section{(S) Supporting Information}

The Supporting Information is available free of charge on the ACS Publications website at DOI: 10.1021/acsomega.6b00320.

Details on the inverse opal structure, degradation, and their behavior (PDF)

\section{AUTHOR INFORMATION}

\section{Corresponding Author}

*E-mail: fiorenzo.omenetto@tufts.edu. Science \& Technology Center, Tufts University, Room 241, Medford, Massachusetts 02155, United States.

ORCID

David L. Kaplan: 0000-0002-9245-7774

Fiorenzo G. Omenetto: 0000-0002-0327-853X

\section{Author Contributions}

P.T. initiated the concept. P.T. and A.N.M. developed the fabrication protocols. P.T. performed the protease experiments. P.T., S.Z., and A.G. performed the cell culture experiments. P.T. and M.B.A. performed the laser-excitation-FLIR studies. P.T. and F.G.O. wrote the manuscript. All authors have given approval to the final version of the manuscript.

\section{Funding}

This work was partially supported by the Office of Naval Research under award N000141310596.

Notes

The authors declare no competing financial interest.

\section{ACKNOWLEDGMENTS}

The authors acknowledge Jeanine Coburn and Rosalyn Abbott for assistance in fibroblast cell and hMSC isolation.

\section{REFERENCES}

(1) McConnell, H. M.; Owicki, J. C.; Parce, J. W.; Miller, D. L.; Baxter, G. T.; Wada, H. G.; Pitchford, S. The Cytosensor
Microphysiometer: Biological Applications of Silicon Technology. Science 1992, 257, 1906-1912.

(2) Pancrazio, J. J.; Whelan, J. P.; Borkholder, D. A.; Ma, W.; Stenger, D. A. Development and Application of Cell-Based Biosensors. Ann. Biomed. Eng. 1999, 27, 697-711.

(3) Spegel, C.; Heiskanen, A.; Skjolding, L. H. D.; Emnéus, J. Chip Based Electroanalytical Systems for Cell Analysis. Electroanalysis 2008, 20, 680-702.

(4) Hug, T. S. Biophysical Methods for Monitoring Cell-Substrate Interactions in Drug Discovery. Assay Drug Dev. Technol. 2003, 1, $479-488$.

(5) Kovacs, G. T. A. Electronic Sensors with Living Cellular Components. Proc. IEEE 2003, 91, 915-929.

(6) Tan, J. L.; Tien, J.; Pirone, D. M.; Gray, D. S.; Bhadriraju, K.; Chen, C. S. Cells Lying on a Bed of Microneedles: An Approach to Isolate Mechanical Force. Proc. Natl. Acad. Sci. U.S.A. 2003, 100, 1484-1489.

(7) Beningo, K. A.; Wang, Y.-L. Flexible Substrata for the Detection of Cellular Traction Forces. Trends Cell Biol. 2002, 12, 79-84.

(8) Balaban, N. Q.; Schwarz, U. S.; Riveline, D.; Goichberg, P.; Tzur, G.; Sabanay, I.; Mahalu, D.; Safran, S.; Bershadsky, A.; Addadi, L.; et al. Force and Focal Adhesion Assembly: A Close Relationship Studied Using Elastic Micropatterned Substrates. Nat. Cell Biol. 2001, 3, 466472.

(9) Charras, G. T.; Horton, M. A. Single Cell Mechanotransduction and Its Modulation Analyzed by Atomic Force Microscope Indentation. Biophys. J. 2002, 82, 2970-2981.

(10) Alvarez, S. D.; Schwartz, M. P.; Migliori, B.; Rang, C. U.; Chao, L.; Sailor, M. J. Using a Porous Silicon Photonic Crystal for Bacterial Cell-Based Biosensing. Phys. Status Solidi A 2007, 204, 1439-1443.

(11) Dancil, K.-P. S.; Greiner, D. P.; Sailor, M. J. A Porous Silicon Optical Biosensor: Detection of Reversible Binding of IgG to a Protein A-Modified Surface. J. Am. Chem. Soc. 1999, 121, 7925-7930.

(12) Blanford, C. F.; Schroden, R. C.; Al-Daous, M.; Stein, A. Tuning Solvent-Dependent Color Changes of Three-Dimensionally Ordered Macroporous (3DOM) Materials Through Compositional and Geometric Modifications. Adv. Mater. 2001, 13, 26-29.

(13) Gupta, B.; Zhu, Y.; Guan, B.; Reece, P. J.; Gooding, J. J. Functionalised Porous Silicon as a Biosensor: Emphasis on Monitoring Cells in Vivo and in Vitro. Analyst 2013, 138, 3593.

(14) Kilian, K. A.; Lai, L. M. H.; Magenau, A.; Cartland, S.; Böcking, T.; Di Girolamo, N.; Gal, M.; Gaus, K.; Gooding, J. J. Smart Tissue Culture: In Situ Monitoring of the Activity of Protease Enzymes Secreted from Live Cells Using Nanostructured Photonic Crystals. Nano Lett. 2009, 9, 2021-2025.

(15) Schwartz, M. P.; Derfus, A. M.; Alvarez, S. D.; Bhatia, S. N.; Sailor, M. J. The Smart Petri Dish: A Nanostructured Photonic Crystal for Real-Time Monitoring of Living Cells. Langmuir 2006, 22, 70847090.

(16) Kim, S.; Mitropoulos, A. N.; Spitzberg, J. D.; Tao, H.; Kaplan, D. L.; Omenetto, F. G. Silk Inverse Opals. Nat. Photonics 2012, 6, 818-823.

(17) Stein, A.; Wilson, B. E.; Rudisill, S. G. Design and Functionality of Colloidal-Crystal-Templated Materials-chemical Applications of Inverse Opals. Chem. Soc. Rev. 2013, 42, 2763-2803.

(18) Kovacs, A.; Jonnalagadda, P.; Meng, X. Y.; Mescheder, U. Characterization of Porous Silicon Based Optical Sensor System for Biosensor Applications. In 2009 IEEE Sensors, 2009; pp 21-26.

(19) Choi, S.-W.; Xie, J.; Xia, Y. Chitosan-Based Inverse Opals: Three-Dimensional Scaffolds with Uniform Pore Structures for Cell Culture. Adv. Mater. 2009, 21, 2997-3001.

(20) Marelli, B.; Omenetto, F. G. Cashmere-Derived Keratin for Device Manufacturing on the Micro- and Nanoscale. J. Mater. Chem. C 2015, 3, 2783-2787.

(21) Swinerd, V. M.; Collins, A. M.; Skaer, N. J. V.; Gheysens, T.; Mann, S. Silk Inverse Opals from Template-Directed $\beta$-Sheet Transformation of Regenerated Silk Fibroin. Soft Matter 2007, 3, $1377-1380$. 
(22) Lu, S.; Wang, X.; Lu, Q.; Hu, X.; Uppal, N.; Omenetto, F. G.; Kaplan, D. L. Stabilization of Enzymes in Silk Films. Biomacromolecules 2009, 10, 1032-1042.

(23) Pritchard, E. M.; Kaplan, D. L. Silk Fibroin Biomaterials for Controlled Release Drug Delivery. Expert Opin. Drug Delivery 2011, 8, $797-811$.

(24) Kasoju, N.; Bora, U. Silk Fibroin in Tissue Engineering. Adv. Healthcare Mater. 2012, 1, 393-412.

(25) Hwang, S.-W.; Tao, H.; Kim, D.-H.; Cheng, H.; Song, J.-K.; Rill, E.; Brenckle, M. A.; Panilaitis, B.; Won, S. M.; Kim, Y.-S.; et al. A Physically Transient Form of Silicon Electronics. Science 2012, 337, 1640-1644.

(26) Kim, D.-H.; Lu, N.; Ma, R.; Kim, Y.-S.; Kim, R.-H.; Wang, S.; Wu, J.; Won, S. M.; Tao, H.; Islam, A.; et al. Epidermal Electronics. Science 2011, 333, 838-843.

(27) Minoura, N.; Aiba, S. I.; Higuchi, M.; Gotoh, Y.; Tsukada, M.; Imai, Y. Attachment and Growth of Fibroblast Cells on Silk Fibroin. Biochem. Biophys. Res. Commun. 1995, 208, 511-516.

(28) Lu, Q.; Wang, X.; Hu, X.; Cebe, P.; Omenetto, F.; Kaplan, D. L. Stabilization and Release of Enzymes from Silk Films. Macromol. Biosci. 2010, 10, 359-368.

(29) Pritchard, E. M.; Dennis, P. B.; Omenetto, F.; Naik, R. R.; Kaplan, D. L. Physical and Chemical Aspects of Stabilization of Compounds in Silk. Biopolymers 2012, 97, 479-498.

(30) Brown, J.; Lu, C.-L.; Coburn, J.; Kaplan, D. L. Impact of Silk Biomaterial Structure on Proteolysis. Acta Biomater. 2015, 11, 212221.

(31) Lu, Q.; Zhang, B.; Li, M.; Zuo, B.; Kaplan, D. L.; Huang, Y.; Zhu, H. Degradation Mechanism and Control of Silk Fibroin. Biomacromolecules 2011, 12, 1080-1086.

(32) Wang, Y.; Kim, H.-J.; Vunjak-Novakovic, G.; Kaplan, D. L. Stem Cell-Based Tissue Engineering with Silk Biomaterials. Biomaterials 2006, 27, 6064-6082.

(33) Tien, L. W.; Gil, E. S.; Park, S.-H.; Mandal, B. B.; Kaplan, D. L. Patterned Silk Film Scaffolds for Aligned Lamellar Bone Tissue Engineering. Macromol. Biosci. 2012, 12, 1671-1679.

(34) Sayin, E.; Baran, E. T.; Hasirci, V. Osteogenic Differentiation of Adipose Derived Stem Cells on High and Low Aspect Ratio Micropatterns. J. Biomater. Sci., Polym. Ed. 2015, 26, 1402-1424.

(35) Diao, Y. Y.; Liu, X. Y.; Toh, G. W.; Shi, L.; Zi, J. Multiple Structural Coloring of Silk-Fibroin Photonic Crystals and HumidityResponsive Color Sensing. Adv. Funct. Mater. 2013, 23, 5373-5380.

(36) Sommer, M. R.; Vetsch, J. R.; Leemann, J.; Müller, R.; Studart, A. R.; Hofmann, S. Silk Fibroin Scaffolds with Inverse Opal Structure for Bone Tissue Engineering. J. Biomed. Mater. Res., Part B 2016, DOI: $10.1002 /$ jbm.b.33737.

(37) Burke, K. A.; Brenckle, M. A.; Kaplan, D. L.; Omenetto, F. G. Evaluation of the Spectral Response of Functionalized Silk Inverse Opals as Colorimetric Immunosensors. ACS Appl. Mater. Interfaces 2016, 8, 16218-16226.

(38) Wang, J. Y.; Cao, Y.; Feng, Y.; Yin, F.; Gao, J. P. Multiresponsive Inverse-Opal Hydrogels. Adv. Mater. 2007, 19, 3865-3871.

(39) Chen, W.; Weng, S.; Zhang, F.; Allen, S.; Li, X.; Bao, L.; Lam, R. H. W.; Macoska, J. A.; Merajver, S. D.; Fu, J. Nanoroughened Surfaces for Efficient Capture of Circulating Tumor Cells without Using Capture Antibodies. ACS Nano 2013, 7, 566-575.

(40) Sengupta, S.; Park, S.-H.; Seok, G. E.; Patel, A.; Numata, K.; Lu, C.-L.; Kaplan, D. L. Quantifying Osteogenic Cell Degradation of Silk Biomaterials. Biomacromolecules 2010, 11, 3592-3599.

(41) Wu, Y.-N.; Law, J. B. K.; He, A. Y.; Low, H. Y.; Hui, J. H. P.; Lim, C. T.; Yang, Z.; Lee, E. H. Substrate Topography Determines the Fate of Chondrogenesis from Human Mesenchymal Stem Cells Resulting in Specific Cartilage Phenotype Formation. Nanomedicine 2014, 10, 1507-1516.

(42) Zheng, Z. Z.; Liu, M.; Guo, S. Z.; Wu, J. B.; Lu, D. S.; Li, G.; Liu, S. S.; Wang, X. Q.; Kaplan, D. L. Incorporation of Quantum Dots into Silk Biomaterials for Fluorescence Imaging. J. Mater. Chem. B 2015, 3, 6509-6519.
(43) Rockwood, D. N.; Preda, R. C.; Yücel, T.; Wang, X.; Lovett, M. L.; Kaplan, D. L. Materials Fabrication from Bombyx Mori Silk Fibroin. Nat. Protoc. 2011, 6, 1612-1631.

(44) Abbott, R. D.; Raja, W. K.; Wang, R. Y.; Stinson, J. A.; Glettig, D. L.; Burke, K. A.; Kaplan, D. L. Long Term Perfusion System Supporting Adipogenesis. Methods 2015, 84, 84-89. 